\title{
Assessment of a procedure for detecting minute levels of tooth erosion
}

\author{
H.L. Mitchell \\ School of Engineering, University of Newcastle, Newcastle, Australia \\ $\&$ \\ R.G. Chadwick \\ S. Ward \\ S.L. Manton \\ The Dental School, University of Dundee, Dundee, UK
}

\section{Corresponding author:}

Dr H L Mitchell,

Discipline of Civil, Surveying and Environmental Engineering,

School of Engineering,

University of Newcastle

$\begin{array}{lll}\text { Callaghan } & \text { NSW } & 2308\end{array}$

Australia

Telephone: $\quad 61-2-49216055$

Fax: 61-2-49216991

Email: harvey.mitchell@newcastle.edu.au 


\begin{abstract}
:
Over a period of some years, the writers have progressively developed the components of a system for discerning erosion on children's teeth, for use in an extensive project seeking correlations between erosion and various perceived risk factors. The detection of minute levels of erosion is to be based on three mappings over an 18 month period of the palatal surfaces of maxillary central incisors of about 250 children. Significant challenges were encountered, the primary problem being the impracticality of placing control marks which would aid the realignment of successive measurements. This paper describes the erosion detection system and the initial experiences based on of the first 100 subjects measured after nine months. The procedures have detected the occurrence of erosion of $50 \mu \mathrm{m}$ magnitude on about one quarter of the teeth over a nine month period, at a precision estimated to be $\forall 15$ $\mu \mathrm{m}$. The occurrence of some anomalous incidents prevented the procedure from being fully automatic, and it necessitated human examination of a graphical diagram derived from the surface matching program, but it was nevertheless superior to current practices of examining impressions or replicas entirely by eye.
\end{abstract}

\title{
Keywords:
}

dentistry, instrumentation, tooth measurement, surface registration 


\section{Introduction}

Tooth surface erosion is a condition that is caused by the action of acids in the mouth to dissolve the teeth, an effect which is to be distinguished from those of decay, attrition and abrasion. According to the 1993 National Survey of Child Dental Health in the United Kingdom (O'Brian, 1994), erosion affects just over half of 5-6 year old children and nearly one quarter of 11 year olds surveyed. If unchecked, erosion, as with dental caries (tooth decay), results in severe tooth destruction necessitating costly and time consuming dental treatment and continual monitoring (Nunn, 1996) throughout life. Both dental caries and erosion arise from the destruction of mineralised tooth tissues by acid, but unlike dental caries, erosion results from direct acid contact with the teeth, and no acid production by the oral microflora is involved. This acid may derive from dietary intake, medication, regurgitation of stomach contents or prolonged exposure to an acidic external environment, (Ten Cate \& Imfeld, 1996). The precise relationship between acidic risk factors and the erosive process is however, unclear for their effects may be modified by both biological (e.g. saliva composition and flow rate, tooth composition and structure) and behavioural (e.g. unusual oral hygiene practices, dieting, eating, drinking and swallowing habits) factors, (Zero, 1996). The problem has lead the writers to embark on a major project aimed at detecting the extent of palatal erosion on children's maxillary central incisors over an 18 month period, with the objective of correlating any identified erosion with various anticipated erosion risk factors, including diet and relevant elements of the children's lifestyle, (Chadwick \& Mitchell, 1998).

The work described here has aimed to improve upon current research methods in this field. In a survey of full time consultants in restorative dentistry (Chadwick, 1998), it was found 
that all currently used study casts of the teeth to subjectively monitor wear. However, one third believed this to be a relatively crude method of detecting wear, capable of distinguishing only gross changes. Two-thirds believed such an approach to lie between crude and highly sensitive, $94 \%$ thought a sensitive quantitative technique would be helpful in identifying when tooth wear exceeded normal physiological limits and $90 \%$ believed such a technique an advance in management. Although a variety of subjective ranking scales have been developed to assess tooth wear (Eccles, 1979; Smith \& Knight, 1984; Oilo et al., 1987) they are insufficiently sensitive to detect small changes in surface topography that would be of assistance in establishing in vivo erosion risk factors. In addition, in vivo research in this area has been severely handicapped by the lack of a sensitive quantitative technique to measure both the level and rate of progression of erosion. Furthermore, the measurement of the erosive process is complicated by the lack of fixed reference points within the oral cavity from which to apply traditional metrological approaches. Some workers have attempted to overcome this problem by creating artificial reference points by either making indentations in enamel (Lambrechts et al, 1984) or by affixing metal discs to the teeth (Bartlett et al., 1997) however, as indentations are subject to the erosive process itself and discs have retention problems they do not solve the problem reliably.

Attempting to detect erosion over the short period of 18 months is therefore ambitious, and the erosion detection system, consisting of a number of disparate components, has to operate within very tight constraints. The writers have been progressively developing and testing the components of the system which has been designed to discern the minute levels of erosion on the children's teeth overcoming the known constraints over a period of some years. The approach involves the mapping of the incisors in conjunction with a mathematical shape comparison, without control points, to detect any erosion. The full project involves the 
measurements of replicas of the palatal surface of the incisors of 250 children, with subsequent analysis to identify erosion which will be correlated with the risk factors as assessed by means of questionnaires. The data available at the time of writing have been processed according to the proposed procedures so it is possible to reflect on the viability of the combination of the component procedures, and to review the feasibility of the erosion detection system as an entity being able to achieve its delicate goals. This paper summarises the outcomes of the investigations of each of the system's components, and, because it is important to assess the system as a whole, the paper reports some initial achievements with the integrated system, taking advantage of some early results obtained from measurements at the nine month epoch to reflect on the possibility of detecting change in the subjects' teeth and make a prediction about the project's likelihood of success. Although the various elements of the total system have been designed and tested during some years of system development, and have each been described via a series of papers prepared over a number of years, in this paper the capabilities of the integrated system are emphasised.

\section{Materials and Methods}

\subsection{Overview}

The subjects were school children aged 10 - 11 years attending four secondary schools within the county of Angus of the United Kingdom. A consent form was issued to all pupils within this age range at each of the schools and all those consenting to participate were included in the study, (except that any subject undergoing orthodontic treatment with a fixed upper appliance or with dental restorations present in the upper central incisors was excluded as this 
would have impaired the necessary impression taking for fabrication of replicas). All children have volunteered to participate in the survey over an 18 month period, and have given informed consent to the taking of appropriate dental castings, and to the completion of a questionnaire relating to their diet and their lifestyle. Ethical approval was obtained from the Tayside Committee on Medical Research Ethics (Ref 040/99). A total of 250 children have been recruited. Staggered starting dates mean that the sampling period extends from early 2001 to late 2002 .

The investigative procedure involves the taking of castings of the children's entire upper dental arch, at 0, 9 and 18 months. From the upper arch castings, replications are then made. The palatal surface of each central incisor is in turn mapped by one of a pair of almost identical electro-mechanical instruments. Differences between the surfaces are then sought in order to define the regions of surface loss which have occurred from one epoch to the next. Because the accurate monitoring of the progression of erosion is complicated by the lack of fixed intra-oral reference points from which to make measurements, the differences between the replicas are estimated in this work by a mathematical surface matching and difference detection algorithm, or SMADDA, the object of which is to estimate the surfaces' best fit position without the aid of control points, and to simultaneously estimate the change which has occurred from one epoch to the next. From the output of a computer program, a judgement is made about the nature and extent of the erosion.

The system's various components are summarised in the following sections of the paper: the creation of impressions and electro-conductive replicas in Section 2.2; the replica measurement hardware in Section 2.3; the computer routines for matching and difference detection in Section 2.4; the system's precision in Section 2.5, and methods of analysis in 
Section 2.6. Correlation of questionnaire answers with any detected erosion has not been carried out yet, and these matters, which are irrelevant to this paper anyway, are not discussed here.

\subsection{Impressions and Replicas}

Impressions of the region of the upper jaw of interest are created using an addition cured silicone impression material (President, Coltène, Switzerland). Fabrication of electroconductive replicas suitable for mapping by the electro-mechanical instrument are carried out by painting the concave surface of impressions with high silver content electroconductive paints (RS Silver Paint, RS Components, Corby, Northants, UK) and, once dry, backing this up with a cyanoacrylate based gel material (Zapit) and die stone.

The choice of the RS Silver Paint was based on an earlier investigation of a number of electroconductive surface coatings on replicas, in which it was determined that RS Silver paint gave replicas that were within $\pm 3 \mu \mathrm{m}$ of the original test piece, (Chadwick et al., 2002a).

\subsection{Replica Mapping}

The mapping instrumentation, developed specifically for measurement within the Dundee Dental School, has been described previously, and the precision of the measurement by the system has been assessed as being of the order of $\pm 5 \mu \mathrm{m}$, (Chadwick et al., 1997).

Manufactured by the Medical Physics Department of Ninewells Hospital, Dundee, UK, to BS EN ISO 9001 (British Standards Institute, 1994), each instrument consists of a translating 
table (Daedal, Pittsburgh, USA), shown at $A$ in Figure 1, motorised by the addition of two computer controlled stepper motors (RS Components Ltd., Corby, UK), B and C in Figure 1, that control precisely the position of the table in the horizontal plane. In addition, a third geared stepper motor, at $D$, under computer control, mounted perpendicular to the motorised table, governs the position of an electrical probe relative to the specimen. The probe was manufactured from tungsten carbide wire of $125 \mu \mathrm{m}$ diameter (Clark Electromedical, Pangbourne, UK) and forms part of a feedback loop such that on coming into close proximity with an electrically conductive tooth replica, seen at $E$, wired into the specimen chamber on the translating table, it ceases its downward travel in the vertical direction and is retracted 50 $\mu \mathrm{m}$ before moving on to the next measurement point. To minimise the effects of backlash in the stepper motors, measurement are only undertaken when the stepper motors were driving the stage in the positive $x$ and $y$ directions. The positioning and measurement resolution in the $x$ and $y$ directions is $+2.5 \mu \mathrm{m}$, and the resolution in the $z$ direction is $1 \mu \mathrm{m}$. Throughout this work the $x$ and $y$ intervals, at which the elevation ( $z$ coordinate) of the object was determined, were set at $150 \mu \mathrm{m}$.

All data is computer logged providing a serial record of coordinates in the horizontal (denoted $x$ and $y$ ) and vertical ( $z$ ) directions. Data files from the measurement of any replica comprise a digital terrain model (DTM) consisting of a set of Cartesian coordinate triplets, i.e. a set of $(x, y, z)$ values, in which the $x, y$ plane can be regarded as being in a direction which is essentially across the incisor surface, while the $z$ reading depicts the surface undulations. Files typically represented a matrix of about 40 points x 40 points, sampled every $150 \mu \mathrm{m}$, giving around 1,600 data points in all to cover a grid of $6 \mathrm{~mm} \times 6 \mathrm{~mm}$. A sample data set created by one measurement of the replica surface is shown at (a) and (b) in Figure 2. 
In order not to transfer any errors of the instruments, each of the replicas for a given subject is mapped using the same instrument.

\subsection{Mathematical surface matching and difference detection}

The SMADDA mathematically registers the two DTMs of the same tooth from different epochs, to determine the degree of surface loss that has occurred. The principles behind the SMADDA and tests of its effective functioning have been reported, (Mitchell \& Chadwick, 1998). The matching algorithm defines the corresponding position of the two surfaces as being that position where the sum of the squares of the surface separations is minimised (assuming certain mathematical solution convergence criteria are met). The surface separation is defined as being measured in the $z$ direction of the co-ordinate system of the first surface; see Figure 3. (If the two surfaces do not differ significantly in rotations about the $x$ and $y$ axes, then the choice as to which surface is regarded as the first or second surface is not significant.) Once the surfaces' best fitting position is located by the surface fitting computer program, it provides the three translation and three rotation transformation parameters which relate the two independent $x y z$ co-ordinate systems of the surface models. Details of the algorithm used for this work have been given previously, (Mitchell \& Chadwick, 1999). It has the same concept as that used by Jovanovski \& Lynch (2000) for similar work.

The surface separation distances after determination of the best fit are the primary source of information, and are used to isolate the erosion. The program operator is able to select the level at which erosion is considered to be identifiable. Moreover, any surface separation distances which are found during processing to be larger than this threshold are excluded 
from influencing the match solution. Furthermore, if the area of excluded surface is a large proportion to the total measured area of tooth (due to, for example, abnormally large areas of erosion or other change in tooth shape), it suggests a solution of unsatisfactory strength, and the match results from that pair of replicas should then not be accepted.

\subsection{Expected Precision}

While it is to be expected that the replication and measurement stages are imperfect, it is not so apparent that the SMADDA program also introduces some errors into the erosion detection. They can be incurred by inexact numerical procedures (notably interpolation and gradient estimation) in the computer program which carries out the surface fitting. They mean that, even with perfectly measured surfaces, some residual surface differences will arise in the surface match. The magnitude of the total random error from mapping and matching has been estimated by using repeated measurements of an electroconductive replica of a maxillary incisor. Ten measurements of the replica were taken with both instruments. The object was moved about $0.1 \mathrm{~mm}$ in the $x$ and $y$ directions between re-measurements, so that the ten sets of co-ordinates mimic the same surface as if sampled on independent occasions when mounted in positions which are not identical, although close. The root-mean-square (RMS) of surface separations obtained from eight comparisons of the ten replica surfaces created from measurements of the same surface when moved slightly, gave a mean of the RMS values of $\pm 10 \mu \mathrm{m}$. Because the random errors were estimated to be $\forall 3 \mu \mathrm{m}$ for the impression and replication procedure (Section 2.2), the overall precision is estimated to be of order $\forall 15 \mu \mathrm{m}$. 


\subsection{Methods of Analysis}

For this work the erosion detection threshold has been set at $50 \mu \mathrm{m}$, being about three times the precision, while the SMADDA wear analysis of any pair of surfaces was rejected if the area of surface above the erosion detection threshold exceeded $33 \%$ of the total area. The SMADDA computer program provides estimates of the erosion as a percentage of the total measured surface area, in bands at $50 \mu \mathrm{m}$ intervals, both positive and negative, for surface separation distances above the $50 \mu \mathrm{m}$ detection threshold, for later analysis of the intensity of the erosion. For each match, the program also provides various statistics (such as whether the solution converged, number of iterations, mean surface separation distance, the mathematical condition of the equations involved in the solution). The statistics are used to help assess whether the match has proceeded satisfactorily.

The information provided by the numerical surface separation distances, however, has been found to be too simplistic for immediate indication of erosion, for various reasons. In particular, a tooth may display multiple regions of depth loss which may deserve to be distinguished from a tooth with a single localised area of erosion, even though the total volume change may be the same. Consequently, the quantitative output was studied in conjunction with the graphical views from that match to provide information about the nature of the locations of detected change. This analysis also revealed the occurrence of some anomalies, which are discussed in Section 3.2. For each match, graphics views of the results were created (Surfer ${ }^{\mathrm{TM}}$ for Windows, Version 6, Golden Software Inc., Colorado, USA) to permit an examination of the distribution and nature of any apparent change. Colour-coded filled contours are used to represent the surface separations in at the $50 \mu \mathrm{m}$ intervals mentioned in the preceding paragraph. See Figure 2. 
The benefits of interpreting erosion from diagrams of this nature have been reported, (Chadwick \& Mitchell, 1999). It is crucial to recognise that, at present, the assessment of progression of palatal surface tooth erosion is made by UK consultants in restorative dentistry by making visual comparison of the dentition with previous study casts, (Chadwick, 1998). The use of graphical views in this study is therefore similar in its concept to a dental practitioner using an optical examination of successive replicas to observe erosion, but the graphical procedure is enhanced by the combined results being given by the SMADDA and by the colour-coding.

In a test of the reliability of the investigators' assessments via quantitative wear data and colour-coded surface representation plots, two examiners independently viewed each colourcoded surface plot as shown in Figure 2, on two separate occasions and categorised the severity of the tooth surface loss on a five point scale according to predefined criteria (Table 1) developed for this work. An overlay template, consisting of a marked square that occupied an area equal to $5 \%$ of the tooth surface, was utilised to determine the proportion of the surface exhibiting tooth surface loss on the colour-coded surface plot. This figure was then used to assign a wear category. The categories allocated by each examiner were subsequently assessed for intra- and inter-examiner agreement. Where disagreement occurred the examiners discussed their findings and reached a consensus on the most appropriate category to allocate. To further test the robustness of this approach a regression analysis of the agreed categorical wear severity score versus the numerically derived percentage of the mapped surface that had undergone change was carried out, (Chadwick et al., 2002b). Results are discussed in Section 3.1. 


\section{Results}

At the time of writing, a total of 106 pairs of replicas (i.e., from 53 participating children) have been measured both at the baseline epoch and nine months later, (and will be measured yet again, after a full 18 months) and have been processed according to the procedures outlined in Section 3.

\subsection{Reliability of Interpretation of Graphical Views}

Of the 106 pairs of electroconductive replicas evaluated to date, a regression analysis of the agreed categorical wear severity score versus the numerically derived percentage of the mapped surface that had undergone change revealed that these two quantities were strongly linearly related (Pearson $r=-0.74$, with $95 \%$ confidence intervals of -0.81 to -0.63 ). It was concluded that the method used to rationalise and interpret the quantitative tooth surface loss data reflects the quantitative data. Its use should be helpful in epidemiological tooth surface loss studies but continued vigilance is required in cases of examiner disagreement.

\subsection{Casting Anomalies}

Examination of graphical views (as described in Section 2.6) indicated that there were occasional major differences between the surfaces. The replicas themselves could be examined in these cases, and in this way the causes of these anomalies were mostly attributable to air-bubbles in impressions and replicas, the inclusion of soft tissue in the measured area (see Figure 2 for an example), occasional sub-standard impression and 
replication (typically where insufficient material at the incisal edge of impression caused breakthroughs), and sometimes poor cleaning of teeth prior to impressions.

\subsection{Procedures}

A summary record of all matches is being maintained, with a note of the percentage of the area occurring as erosion at different in the different quantities. However, the graphical views are used for the final decision about the extent of erosion.

Although the use of graphical views permitted the occurrence of anomalies to be exposed, and help in the filtering of outlier regions from surface separations, it was not seen as necessary to edit the anomalies from the data, as the anomalies were not seen as affecting the decisions, provided that the graphics views were used during the judgment stages.

An electronic copy of the relevant diagram for each match was retained, with a unique filename.

\subsection{Erosion Detection}

The 106 available pairs of replicas have been processed following the judgement criteria enumerated in Table 1, which also shows the results. The results are based on one of the assessors only, for simplicity. The table shows that 28 teeth exhibited erosion, compared with 78 which were not classified as showing erosion, or approximately one quarter of the teeth were judged to be exhibiting apparent erosion of a magnitude of around $50 \mu \mathrm{m}$ or greater 
which has occurred over a nine month period. On occasions, some imperfections in the matching procedure lead to the depiction of gains as well as losses in tooth surface.

\subsection{Replicas Without Erosion}

In some cases, no differences above the threshold between the two replications were determined. These teeth were then judged to be erosion free. The mean of all RMS differences from such cases was about $15 \mu \mathrm{m}$, supporting the precision results given at section 2.6.

\section{Discussion}

This paper firstly is a description of the components of a system designed to detect minor changes in tooth surface, but secondly and more importantly, it provides an assessment of the system's performance in the early stages of erosion study. The first uses of the system have revealed there are events which prevent the procedure from being fully automatic, as had been initially planned: occasional anomalous outcomes arise from imperfect casting and/or replication and by the existence of excessive areas of erosion or other change which prevent the mathematical algorithm from yielding a usable outcome. The consequences are a need to check each result by human inspection, assisted by an examination of the original replicas as necessary or desired. This may appear to reduce the procedure to current practice of simple examination of impressions or replicas by eye, but it has to be recognised that the mapping and matching process provides a colourful graphic view of the mathematically overlaid specimens, and thus it significantly facilitates the inspection procedure. On the other hand, 
the process offered by the writers does require access to relative expensive mapping hardware and surface matching software which is not commonly available. Nevertheless it is superior to current practice, and indeed this work demonstrates the difficulties and dangers of that practice.

A prediction that the system would detect erosion had come from separate tests of the various components. The system described here has been able to detect change at the $50 \mu \mathrm{m}$ level, a threshold which was found to provided an acceptable and convenient match rejection rate. The procedures appear to have a precision approaching $\pm 15 \mu \mathrm{m}$, according to both repeat tests on the same replica or teeth which appear to suffer from no erosion. It is foreseeable that, as experiences are extended, procedures may be refined to reduce the magnitude of the current $50 \mu \mathrm{m}$ erosion distinguishing level closer to the estimated $\pm 15 \mu \mathrm{m}$ precision level. Because annual wear rates for enamel in normal physiological function are quoted in the literature to be of the order of 10 to $30 \mu \mathrm{m}$ (see Lambrechts et al., 1989; Pintado et al., 1997) it might be assumed that erosion, as an abnormal occurrence, would cause surface loss greater than this, so that the detection of surface change at magnitudes of $50 \mu \mathrm{m}$ are seen as reasonable goals for this work.

To date, tooth surface losses at the $50 \mu \mathrm{m}$ level, even over a short nine month period, have been detected. The results of each match have been checked by an examination of the SMADDA statistics in conjunction with the graphical output. As a result of the initial experiences, the writers have been able to refine the procedures which are to be implemented for the remaining stages of the project, including the measurement of all replicas at the 18 month epoch. 


\section{Acknowledgements}

The work reported in this paper was part of an ongoing project, which seeks to investigate the relationship between palatal dental erosion and dietary variables. The project has been funded by the Chief Scientist Office of the Scottish Executive (Grant Number $\mathrm{K} / \mathrm{MRS} / 50 / \mathrm{C} 2734$ ), but the views expressed are those of the authors. 


\section{References}

Bartlett, D.W., Blunt, L. \& Smith, B.G.N., 1997. Measurement of tooth wear in patients with palatal erosion, British Dental Journal, 182, 179-184.

British Standards Institute, 1994. 'BS EN ISO 9001:1994 Model for quality assurance in design, development, production, installation and servicing', British Standards Institute, London, UK.

Chadwick, R.G., 1998. Toothwear - towards the third dimension, Bulletin of the Royal College of Physicians and Surgeons of Glasgow, 27, 31-32.

Chadwick, R.G. \& Mitchell, H.L., 1998. Conduct of an algorithm in quantifying simulated palatal erosion. Abstract No. 14, J. Dent. Res., 77 (Special Issue B), 633.

Chadwick, R.G. \& Mitchell, H.L., 1999. Presentation of quantitative tooth wear data to clinicians, Quintessence International, 30, 393-398.

Chadwick, R.G., Mitchell, H.L., Cameron, I., Hunter, B., \& Tulley, M., 1997. Development of a novel system for assessing tooth and restoration wear, J. Dent., 25, 41-47.

Chadwick, R.G., Mitchell, H.L. \& Ward, S., 2002a. Evaluation of the accuracy and reproducibility of a replication technique for the manufacture of electroconductive replicas for quantitative clinical wear studies, J. Oral Rehab., 29, 540-545. 
Chadwick, R.G., Mitchell, H.L. \& Ward, S., 2002b. Rationalisation of quantitative tooth surface loss data for epidemiological research, accepted July 2002, J. Oral Rehab.

Eccles, J.D., 1979. Dental erosion of non industrial origin: A clinical survey and classification, Journal of Prosthetic Dentistry, 42, 649-653.

Jovanovski, V. \& Lynch E., 2000. Analysis of the Morphology of oral structures from 3-D co-ordinate data. In: Faller R.V. ed., 'Assessment of Oral Health Diagnostic Techniques and Validation Criteria', Monographs in Oral Science, Volume 17, Mason, Ohio, Karger.

Lambrechts, P., Braem, M., Vuylsteke-Wauters, M. \& Vanherle, G., 1989. Quantitative in vivo wear of human enamel, J. Dent. Res., 68, 1752-1754.

Lambrechts, P., Vuylsteke-Wauters, M., Vanherle, G. \& Davidson, C.L., 1984. Quantitative evaluation of the wear resistance of posterior dental restorations. A new three dimensional measuring technique, Journal of Dentistry, 12, 252-267.

Mitchell, H.L. \& Chadwick, R.G., 1998. Mathematical shape matching as a tool in tooth wear assessment: development and conduct, J. Oral Rehab., 25, 921-928.

Mitchell, H.L. \& R.G. Chadwick, 1999. Digital photogrammetric concepts applied to surface deformation studies, Geomatica, 53(4), 405-414.

Nunn, J.H., 1996. Prevalence of dental erosion and the implications for oral health, European Journal of Oral Sciences, 104, 156-161. 
O'Brian, M., 1994. 'Children's Dental Health in the United Kingdom 1993', Office of Population Censuses and Surveys, Her Majesty's Stationery Office, London.

Oilo, G., Dahl, B.L., Hatle, G. \& Gad, A., 1987. An index for evaluating tooth wear, Acta Odont. Scand., 45, 361-365.

Pintado, M.R., Anderson, G.C., De Long, R. \& Douglas, W.H., 1997. Variation in tooth wear in young adults over a two-year period, Journal of Prosthetic Dentistry, 77,313-320.

Smith, B.G.N. \& Knight, J.K., 1984. An index for measuring the wear of teeth, British Dental Journal, 156, 435-438.

Ten Cate, J.M. \& Imfeld, T., 1996. Dental erosion (Summary), European Journal of Oral Sciences, 104, 241-244.

Zero, D.T., 1996. Etiology of dental erosion - extrinsic factors, European Journal of Oral Sciences, 104, 162-177. 
Table 1: The criteria used to categorise the severity of wear as indicated on colour-coded surface difference plots generated by quantitative wear analysis, and the results of assessing 53 matches of pairs of replicas, according to one assessor, using the criteria.

\begin{tabular}{|c|c|c|}
\hline Category & Criteria & Number \\
\hline 1 & $\begin{array}{l}\text { Majority of surface unchanged with } 5 \% \text { or } \\
\text { less exhibiting tooth surface loss. } \\
\text { (Uncertain cases between categories } 1 \text { and } \\
2 \text { have been categorised as showing no } \\
\text { change.) }\end{array}$ & 78 \\
\hline 2 & $\begin{array}{l}\text { Majority of surface unchanged with } 6-15 \\
\% \text { exhibiting tooth surface loss. }\end{array}$ & 22 \\
\hline 3 & $\begin{array}{l}\text { Majority of surface unchanged with } 16- \\
25 \% \text { exhibiting tooth surface loss. }\end{array}$ & 4 \\
\hline 4 & $\begin{array}{l}26-50 \% \text { of the surface exhibits tooth } \\
\text { surface loss. }\end{array}$ & 2 \\
\hline 5 & $\begin{array}{l}51 \% \text { or greater of the surface exhibits } \\
\text { tooth surface loss. }\end{array}$ & 0 \\
\hline & $\begin{array}{l}\text { Result rejected (cases in which the need } \\
\text { for rejection is uncertain are rejected) }\end{array}$ & 17 \\
\hline
\end{tabular}




\section{Figure captions:}

Figure 1: Photograph of one of the measuring instruments. Lettered items are referred to in the text.

Figure 2: Pictorial representation of a data set derived from the measurement of the replica incisor surface and the match results. The orientation is the same as for Figure 2. The three figures show the measured replica surface at the first epoch (top), the second epoch (middle), and the first surface redrawn with determined differences overlaid in a coded scale, the originals being in colour (lower diagram). The diagram is created with 50 micron contours, with different colours per contour. The last diagram also shows an area of tooth surface loss at the far side, and that inclusion of some soft tissue has caused an apparent surface growth in the lower right corner, and indicated by the dark colouring.

Figure 3: Pictorial representation of a profile of a sample replica surface measurement. Use of line segments illustrate that surfaces are defined as a series of point measurements. 


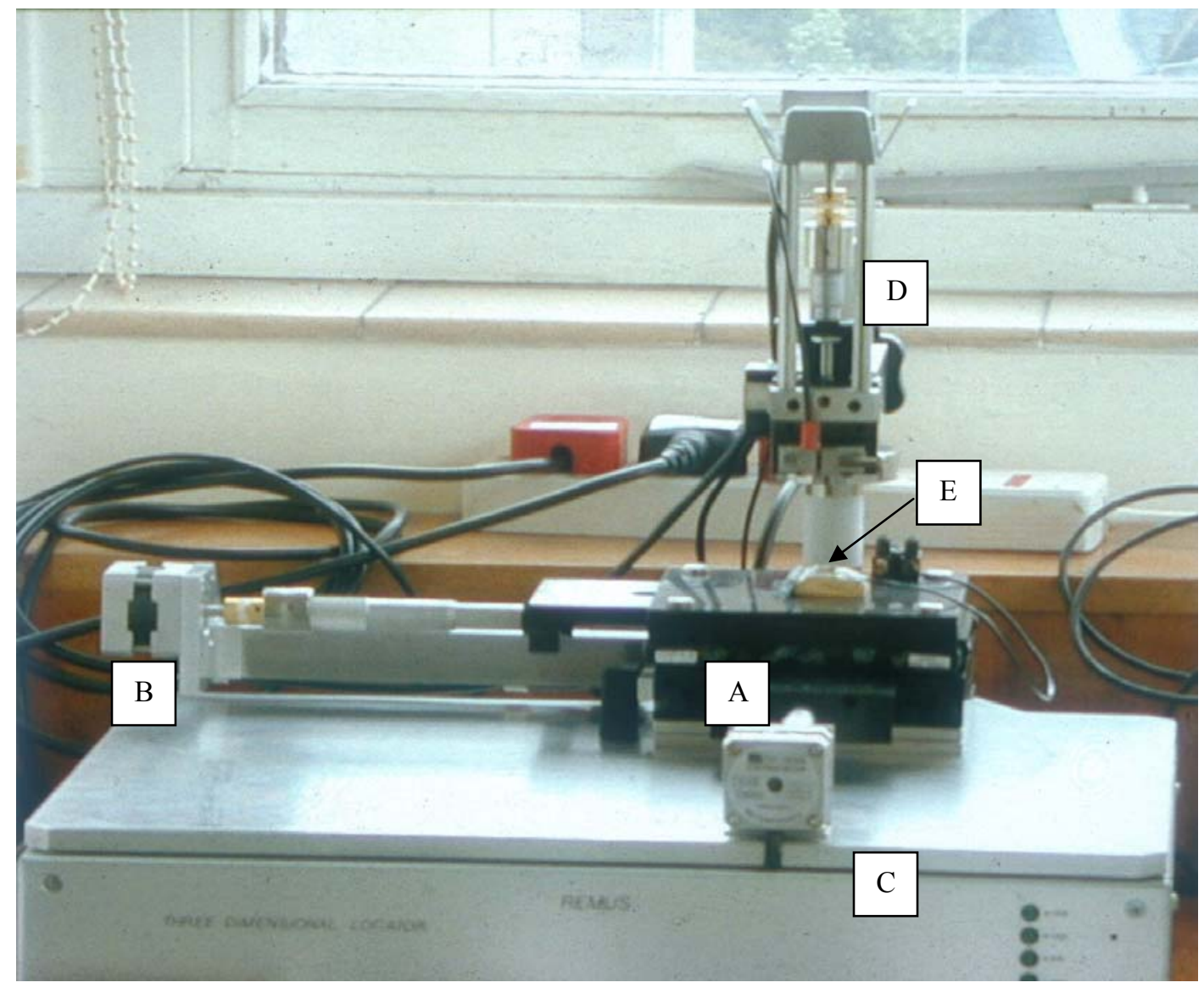




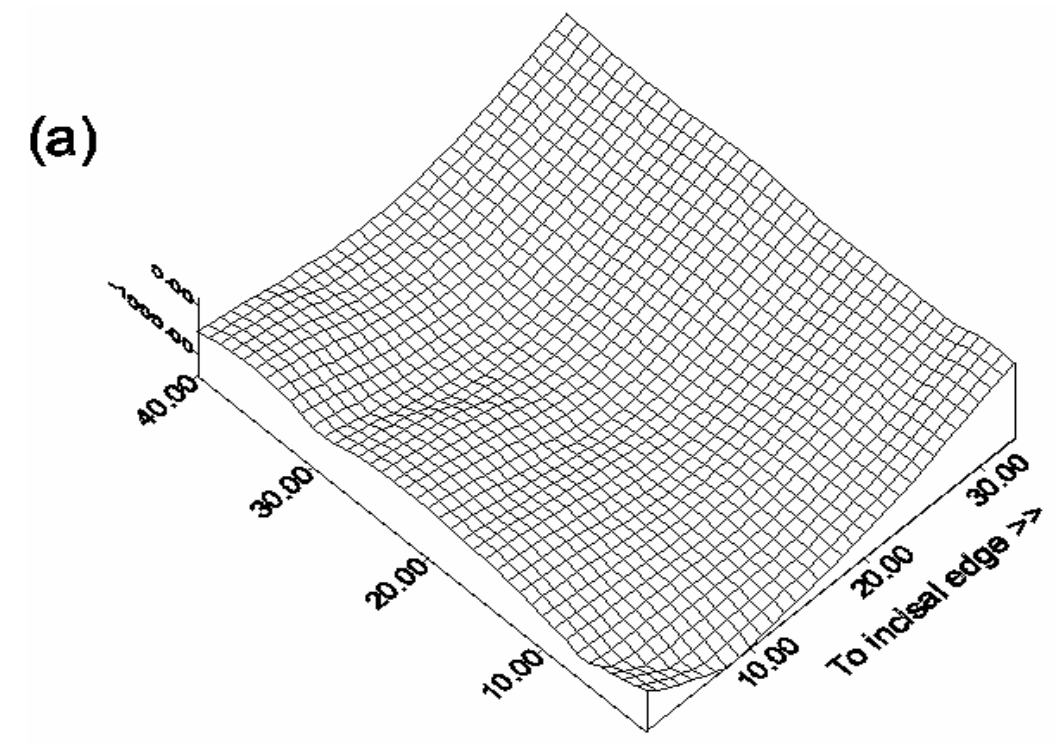

(b)

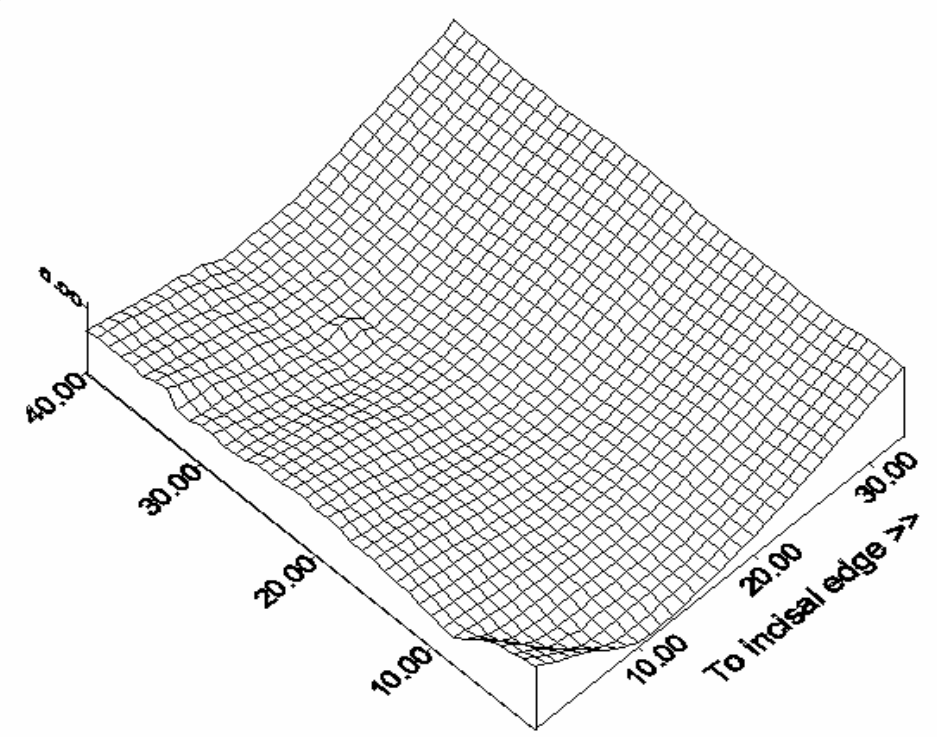

(c)

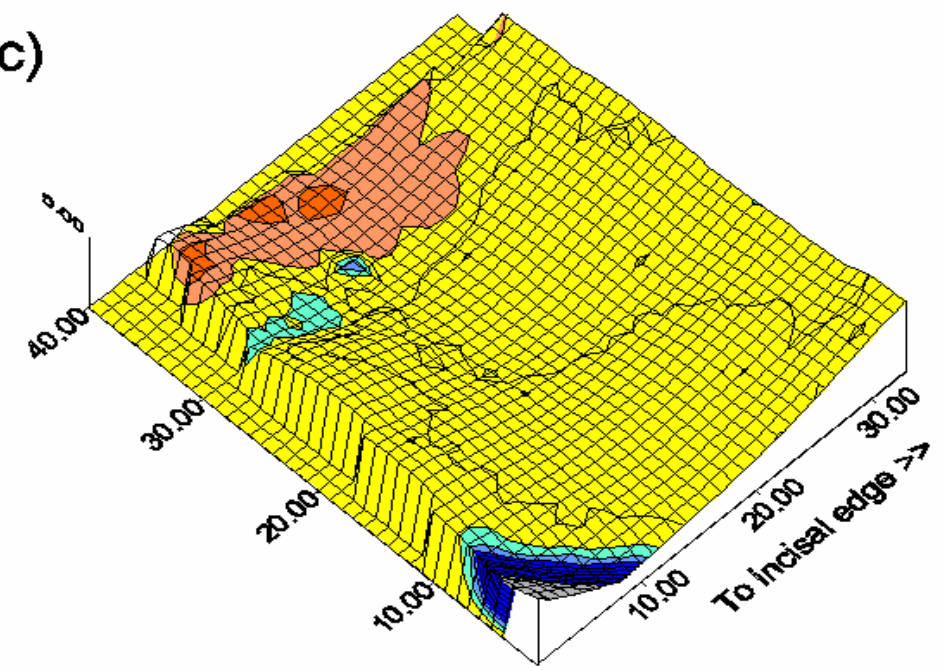




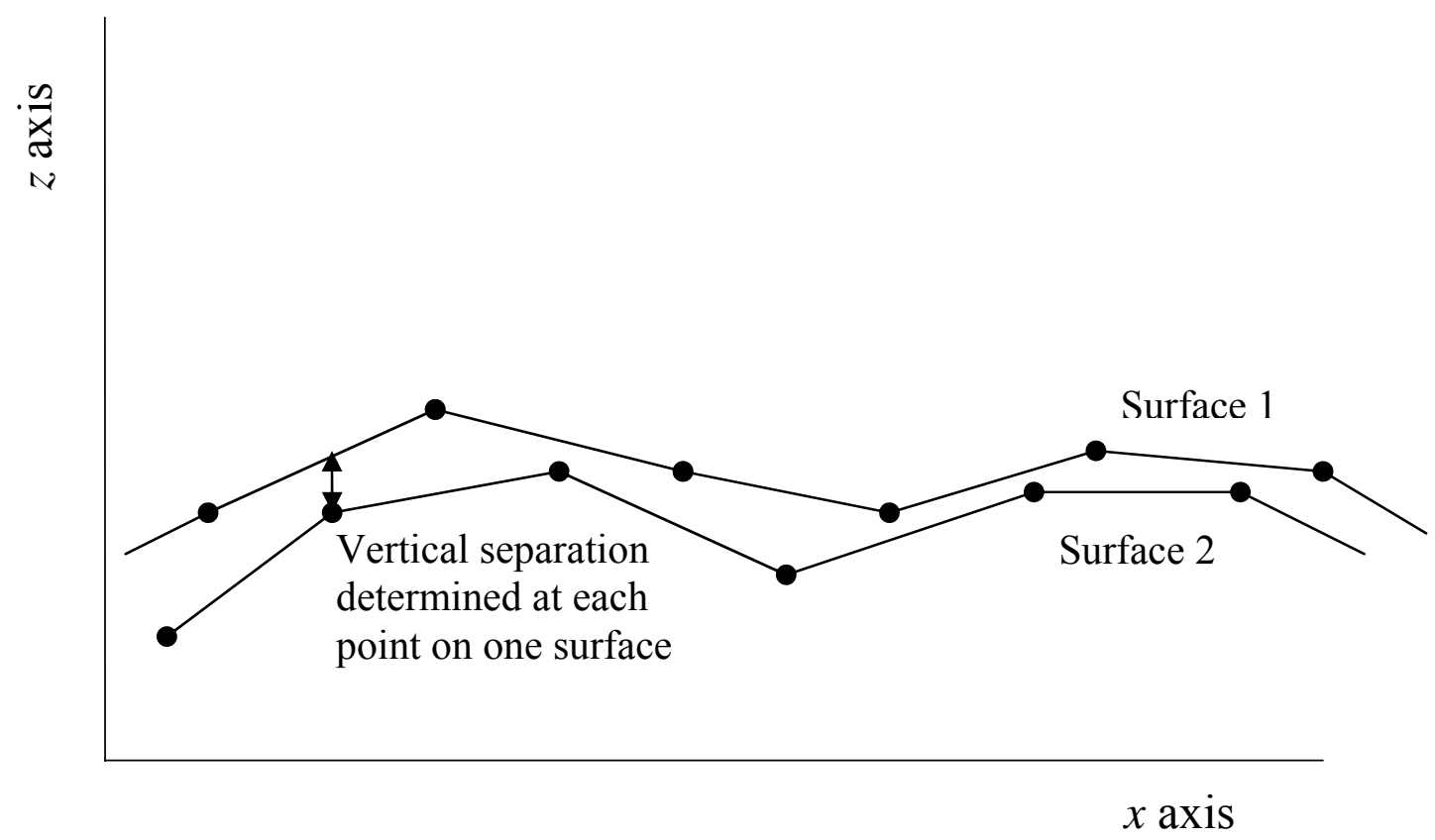

